\title{
Assessment of clinical and therapeutic factors in patients with nasopharyngeal undifferentiated carcinoma
}

\section{Helma Maria Chedid ${ }^{1}$, Sergio Altino Franzi ${ }^{2}$, Rogério Aparecido Dedivitis ${ }^{3}$}

Keywords: nasopharynx, carcinoma, chemotherapy, radiotherapy.

\section{Summary}

\begin{abstract}
$\mathrm{T}$ opharyngeal carcinoma (NPC) is a rare cance with a high incidence in Southern Asia. Aim: to study the demographic, clinical, therapeutic, and prognostic factors of nasopharyngeal undifferentiated carcinoma in a reference service. Materials and methods: A retrospective study was made of 46 patients from January 1998 to August 2000. The patients had no previous treatment and did not present any evidence of synchronous tumors or distance metastases. Results: The age ranged from 14 to 78 years (mean = 46 years); 35 (76\%) patients were male. All patients were Caucasian or African-Brazilian. The onset of initial symptoms ranged from 1 to 48 months (mean $=7$ months); $47 \%$ of the subjects smoked tobacco and 33\% consumed alcoholic beverages. A lump in the neck was the most frequent symptom (34 patients). Twenty-two patients were clinically staged as T1/T2 and 24 patients as T3/T4; 24 patients were classified as N2, and 16 patients were staged as N3. Curative treatment consisted of radiotherapy and simultaneous chemotherapy in clinical stages III and IV. Of 27 patients that were monitored, $52 \%$ were alive with no evidence of disease after three years. Conclusion: All patients were in advanced clinical stages of the disease. The three-year disease-free survival rate was $52 \%$.
\end{abstract}

\footnotetext{
${ }^{1}$ Specialist in Head \& Neck Surgery, Master's degree student in Health Sciences, Heliopolis Hospital - Hosphel, Sao Paulo. Surgeon of the Head \& Neck Surgery and Otorhinolaryngology Department, Heliopolis Hospital - Hosphel, São Paulo.

${ }^{2}$ Doctor in Medicine, graduate course in Oncology, Medical School, Sao Paulo University. Faculty member in the graduation course Health Sciences, Heliopolis Hospital - HOSPHEL, So Paulo.

${ }^{3}$ Doctor in Medicine, graduate course in Otorrinolaringologia and Head \& Neck Surgery, UNIFESP - Paulista School of Medicine. Faculty member of the graduation course in Health Sciences, Heliopolis Hospital - HOSPHEL, Sao Paulo.

Full Professor, Otorrinolaringologia and Head \& Neck Surgery, Santos Metropolitan University. Full Professor, Head \& Neck Surgery Department, Fundacao Lusiada, Santos.

Graduation Course in Health Sciences, Heliopolis Hospital - HOSPHEL, São Paulo.

Address for correspondence: Rua Conego Xavier 276 Sao Paulo SP 04231-030.

This paper was submitted to the RBORL-SGP (Publishing Manager System) on 1 April 2007. Code 3950.

The article was accepted on 22 July 2007.
} 


\section{INTRODUCTION}

The incidence of nasopharyngeal carcinomas is rare in most of the world, except among Southeastern Asian populations, where it is one of the most frequent malignancies. The highest incidences are seen in Alaskan and Greenland Eskimos and in Tunisia. The incidence remains high in Asians who have migrated to Western countries, compared to Caucasians. ${ }^{1,2}$ The etiology is still unknown. There are a few hypotheses, such as hereditary causes related to the HLA-A2 and HLA-Bsin ${ }^{2}$ viral genotypes, Epstein-Barr virus (EBV) infection, altered chromosomes and cultural factors such as increased amounts of fish and preserved food consumption that are rich in nitrosamides, known environmental carcinogens. ${ }^{3,4}$ The EBV may be found in tumor cells of patients by an RNA signal, 5 suggesting that peripheral blood may be investigated in endemic areas of this disease to detect anti-EBV antibodies. As opposed to other head and neck malignancies, smoking and alcohol consumption appear to be less important in the carcinogenesis of the aforementioned tumors. The prognosis of nasopharyngeal tumors depends on initial clinical staging and the histological subtypes. ${ }^{3}$

The incidence is distributed across all age groups, predominating at 50 and 60 years; males are affected more often. The incidence reaches 10 to 20 per 100 thousand male inhabitants and 5 to 10 per 100 thousand females in certain Southeastern regions of China. ${ }^{6}$

The diagnosis tends to be made when the disease is advanced, including signs and symptoms of invasion of the cranial base and cranial nerve involvement. ${ }^{7}$ Symptoms in early disease are rare, and may include nasal block, headaches, deafness and otitis media. In later phases of the disease, invasion of adjacent structures to the cranial base affect more often the maxillary branch of the trigeminal nerve and the abducens nerve. ${ }^{7}$

The incidence of neck metastases upon the initial presentation is high, and may be the first sign of the disease in $44 \%$ to $57 \%$ of cases. Bilaterally involved neck lymph nodes are found in 50\% of cases of undifferentiated carcinomas. ${ }^{8}$ A study of over 5,000 nasopharyngeal carcinoma patients showed that 29\% had distance metastases and 17\% of these presented lesions exclusively in distant sites.

The initial treatment of choice is radiotherapy for tumors at early stages, and radiotherapy with chemotherapy for tumors at advanced stages. ${ }^{10,11}$ Salvage surgery is done for the removal of large neck metastases. Salvage surgery in persistent primary tumors or recurring tumors is rarely indicated.

The purpose of this study was to assess the oncological results of treating nasopharyngeal carcinomas during a 20-year period in a reference head and neck unit.

\section{SERIES AND METHOD}

This was a retrospective study that surveyed the medical files of 46 patients diagnosed histopathologically with undifferentiated nasopharyngeal carcinomas between January 1978 and August 2000. The Research Ethics Committee of the institution approved the study (number 550).

Inclusion criteria were patients with untreated disease, a confirmed histopathological diagnosis, absence of other synchronic neoplasms, absence of distance metastases upon the initial presentation, and initial therapy with curative intent. Between 1978 and 1985 undifferentiated carcinomas were diagnosed by histopathology, because immunohistochemical testing only became available at the institution from 1985 onwards.

The diagnosis of disease was made based on locoregional examination and exams such as nasofibrolaryngoscopy and computed tomography of the face. The definite diagnosis of all cases of undifferentiated nasopharyngeal carcinomas was made by the pathological examination (HE staining) and immunohistochemistry of biopsies of nasopharyngeal lesions, taken by posterior rhinoscopy or nasosinusal endoscopy.

Teletherapy (cobalt unit and linear accelerator) was used for radiotherapy. The radiation dose ranged from 65 to $75 \mathrm{~Gy}$ on the primary tumor, divided into $1.8 \mathrm{~Gy} / \mathrm{day}$, five days a week. Neck lymph node chains received at least 45 Gy to $50 \mathrm{~Gy}$.

Simultaneous chemotherapy was done with cisplatin (CDDP), $100 \mathrm{mg} / \mathrm{m} 2 /$ week, infused intravenously in one hour. Chemotherapy was given by the arterial route in one case.

The following variables were assessed: age of onset of disease, race, clinical presentation at the time of the diagnosis, duration of the clinical history between the first symptom or sign and the first visit to a specialist, the initial therapy, local and regional recurrences, and distance metastases.

\section{RESULTS}

The age of patients treated for undifferentiated nasopharyngeal carcinomas ranged from 14 to 78 years (mean 46 years). There were 35 male patients $(76 \%)$ and 11 female patients (23\%). All patients were either Caucasian or mixed color/black. There were no patients of Asian origin.

The mean time elapsed between the first symptoms and the first visit to a specialist was seven months ( 1 to 48 months). There were $47 \%$ smokers and $33 \%$ users of alcoholic beverages.

The most commonly reported symptoms were the presence of a lump in the throat (34 patients), followed by 
nasal bleeding (10 patients), nose block (seven patients), and pain and weight loss (three patients).

Clinical staging was based on the International Tumor Classification (AJC-UICC); all 46 patients were restaged according to the latest revision done in 2002. Relative to the primary tumor, 10 cases were T1; 12 cases were T2; eight cases were T3, and 16 cases were T4. Relative to neck metastatic lymph nodes, five patients were N0, seven patients were N1, nine patients were N2, and 25 patients were N3. Clinical staging (CS) showed that no patient was EC I or II, 16 patients were EC III, and 30 patients were EC IV. The locoregional exam showed that 13 patients (28.2\%) presented lesions on the nasopharyngeal roof. In 24 patients $(52.1 \%)$, lesions were on the lateral walls. In nine patients (19.5\%), the primary lesion was not located in the medical examination, being diagnosed by image exams.

The physical examination suggested an advanced primary tumor with cranial base or intracranial invasion in seven patients (15.2\%). Paralysis of the 6th cranial nerve was seen in three patients (6.5\%); paralysis of the 9th cranial nerve was seen in two patients (4.3\%); and paralysis of the 10th cranial nerve was seen in two patients (4.3\%). There was no paralysis in more than one cranial never in these seven patients.

Therapy in all patients was conventional external radiotherapy. Concomitant chemotherapy was done in six patients (13\%). Chemotherapy was given by the intra-arterial route in one patient (16.6\%); the remaining five patients (83.4\%) received systemic intravenous chemotherapy.

The radiotherapy dose on the primary tumor ranged from 60 to $70 \mathrm{~Gy}$; the radiotherapy dose on neck lymph node chains ranged from 34 to $50 \mathrm{~Gy}$. An extra dose (boost) was done postoperatively on the dissected surgical bed in three of four patients that underwent neck salvage surgery.

Follow-up was done for 27 (58.7\%) of 46 patients. Of these 27 patients, four (14.8\%) underwent neck salva-

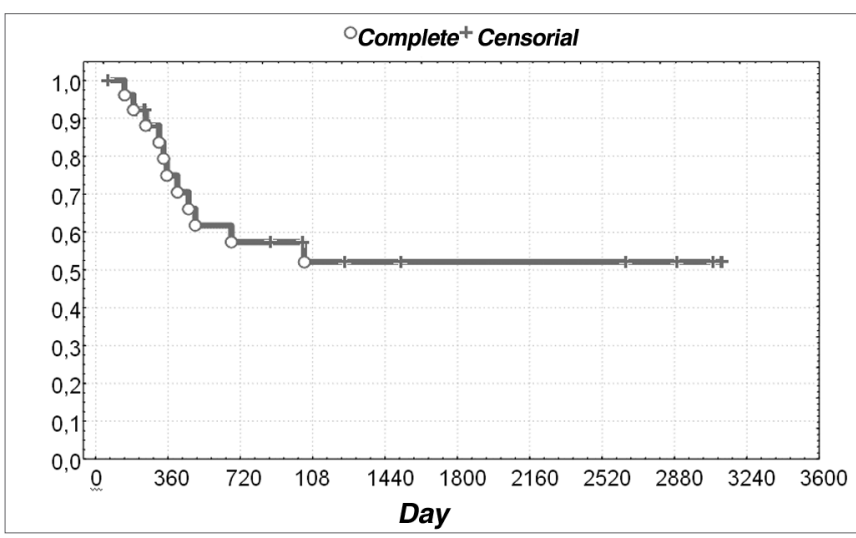

Figure 1. Distribution of disease-free survival of patients with undifferentiated nasopharyngeal carcinomas. ge surgery due to persistent or recurrent palpable neck lymph nodes.

Distance metastases were diagnosed in seven patients (25.9\%) during a 296 month follow-up period. There was locoregional control of the disease and involvement of the brain, bones, lungs and skin.

Of the 27 patients that were followed-up after the initial treatment, $52 \%$ were alive and disease-free at three years. The disease-free survival rate was 52\% (Figure 1).

\section{DISCUSSION}

According to the World Health Organization, nasopharyngeal carcinomas may be divided into three types, as follows: type 1 - epidermoid carcinomas, which occurs mostly in adults; type 2 - non-keratinized carcinomas; and type 3 - undifferentiated carcinomas. Type 1 occurs in less than $5 \%$ of cases in endemic areas, and in $25 \%$ of patients in non-endemic areas. Type 1 is also the least sensitive to radiotherapy; its prognosis is the worst. ${ }^{8}$

In our study most of the patients were in the fifth decade of life. There was no bimodal distribution (a first peak in adolescents and young adults). Race was a second variation compared to the literature; most of the patients were Caucasian, and there were no patients of Asian origin. ${ }^{12,13}$ These findings coincide with those of the National Cancer Institute (USA), showing a higher incidence of nasopharyngeal carcinomas in young adult Caucasians and Afro-Americans compared to Asian patients. ${ }^{14}$

Tobacco and alcohol are relevant etiological factors in upper digestive airways. Undifferentiated nasopharyngeal malignancies, however, are less affected by these substances, which are not considered etiological in these cases. ${ }^{15}$

Radiotherapy and chemotherapy are the preferred therapies in nasopharyngeal carcinomas. Radiotherapy is the main treatment in initial tumors. Chemotherapy is added in cases with advanced tumors. ${ }^{10,16}$

Computed tomography and magnetic resonance imaging currently have a significant role when planning radiotherapy for nasopharyngeal tumors. A study of 275 magnetic resonance exams showed that there was extension of metastases to the retropharynx in $63.6 \%$ of cases, ${ }^{17}$ which is relevant when planning therapy.

The side effects of radiotherapy have decreased with the advent of other forms of irradiation (brachytherapy and stereotaxic radiotherapy) compared to conventional external radiotherapy. The most frequent side effects are xerostomy, otitis media, trismus and severe neck fibrosis. $^{18}$

A randomized study of 229 patients with advanced stage tumors showed that radiotherapy alone or with chemotherapy did not improve local and regional control indices. ${ }^{19}$ Another randomized trial from the Intergroup Study 0099 demonstrated improved disease remission rates 
by using chemotherapy with radiotherapy in advanced stage tumors; in this study, 3-year disease-free survival was $69 \%$ compared to $24 \%$ when treated with radiotherapy alone. ${ }^{10}$

The Asian Oceanian Clinical Oncology Association found no benefits in using neoadjuvante chemotherapy with radiotherapy in advanced nasopharyngeal carcinomas. They did find a lower incidence of local and regional recurrences, but no change in the disease-free survival. ${ }^{20}$

The literature on control of this disease presents mixed results. The first published studies had control rates of $87 \%$ in $\mathrm{T} 1,94 \%$ in $\mathrm{T} 2,68 \%$ in $\mathrm{T} 3$, and $44 \%$ in $\mathrm{T} 4$ cases relative to the primary tumor. ${ }^{21}$ Later papers showed improved control rates for advanced tumors, with $73 \%$ and $100 \%$ in $\mathrm{T} 3$, and $71 \%$ and $63 \%$ in $\mathrm{T} 4 .{ }^{22,23}$ For the N stage, disease control rates are similar in a number of studies, reaching $92 \%$ and $90 \%$ for $\mathrm{N} 0,87 \%$ and $88 \%$ for $\mathrm{N} 1$, and $89 \%$ and $82 \%$ for $\mathrm{N} 3.22,23$

The incidence of local recurrence ranges from 18\% to $58 \%$. Therapy consists of reirradiation or salvage surgery, the choice of which remains controversial. Reirradiation implies in more complications such as cerebral necrosis, hypothalamic-pituitary failure, deafness, retinopathy, transverse actinic myelitis and cranial nerve dysfunction. There have been fewer complications by using brachytherapy and stereotaxic radiotherapy. Control of local recurrence with a second round of radiotherapy has been reported in $10 \%$ to $30 \%$ of cases. ${ }^{24}$

Regional recurrence rates vary from $8 \%$ to $34 \%$ of cases, similar to the cases of lymph node disease persistence at the end of therapy. In these cases, salvage neck dissection is the main form of treatment; success rates reach $66 \%$ of cases. Even with regional disease control, patients tend to course with distance metastases. ${ }^{8,23}$

Neck dissection following the initial therapy used to be recommended in all cases of pre-radiotherapy extensive lymph node disease. 25 In a series with 27 patients that underwent programmed neck dissection, all cases had positive lymph nodes in pathology; $84 \%$ of cases had extracapsular rupture.

The incidence of hematogenic metastases is high; they commonly occur following locoregional disease control. Metastases at a distance arise in $5 \%$ to $10 \%$ of patients upon the diagnosis. Frequent sites include the lungs, bones and liver.26 A study of 900 patients demonstrated metastases at a distance in $22.2 \%$ of cases during the follow-up period of patients that had local and regional disease control. ${ }^{27}$

The treatment of choice in our unit is radiotherapy alone in stages I and II, and radiotherapy with chemotherapy in stages III and IV. In this paper only six patients were also treated with chemotherapy in advanced disease. During the timeframe of this study, radiochemotherapy for nasopharyngeal carcinomas in clinical stages III and
IV was recommended as the ideal treatment; this measure eventually became the therapeutic guideline. In cases with large unilateral or bilateral neck metastases, in which planning radiotherapy is difficult, neoadjuvante chemotherapy is used from 1 to 3 cycles to reduce the size of such metastases, after which radiochemotherapy is done. The indication of neoadjuvante chemotherapy is based on some papers in the literature that have demonstrated its benefits; in such cases, toxicity is low and there is an increase in disease-free survival..$^{28-29}$

Neck surgery is done if lymph nodes persist, if there are large metastases with probable extracapsular extravasation that responded to therapy with complete clinical remission, in salvage of cervical lymph nodes (especially in $\mathrm{N} 3$ cases) and in salvage neck dissection following recurrences.

\section{CONCLUSION}

The distribution of undifferentiated carcinoma did not follow a bimodal distribution per age group. All of the treated patients were in advanced clinical stages (III and IV), and had a 52\% disease-free survival rate at three years.

\section{REFERENCES}

1.Cooper JS, Cohen R, Stevens RE. A comparison of staging systems for nasopharyngeal carcinoma. Cancer 1998;83:213-9.

2.Dickson RI. Nasopharymgeal carcinoma: an evaluation of 209 patients Laryngoscope 1981;91(3):333-54.

3.Marks JE, Philips JL, Menck HR. The national cancer data base report on the relationship of race and national origin to the histology of nasopharyngeal carcinoma. Cancer 1998;83:582-8.

4.Farrow DC, Vaughan TL, Berwick M, Lynch CF, Swanson CM, Lyon JL. Diet and nasopharyngeal cancer in a low-risk population. Int J Cancer 1998;78:675-9.

5.Wei WI, Sham JS. Nasopharyngeal carcinoma. Lancet 2005;365(9476):204154 .

6.Ho JHC. Nasopharyngeal carcinoma. Adv Cancer Res 1972;15:57-92.

7.Turgut M, Erturk O, Saygi S, Ozcan OE. Importance of cranial nerve involvement in nasopharyngeal carcinoma. A clinical study comprising 124 cases with special reference to clinical presentation and prognosis. Neurosurg Rev 1998;21(4):243-8.

8. Brennan B. Nasopharyngeal carcinoma. Orphanet J Rare Dis. 2006;26:123.

9.Lee AW, Poon YF, Foo W, Law SC, Cheung FK, Chan DK et al. Retrospective analysis of 5037 patients with nasopharyngeal carcinoma treated during 1976-1985: overall survival and patterns of failure. Int J Radiat Oncol Biol Phys 1992;23(2):261-70.

10. Al-Sarraf M, LeBlanc M, Giri PG, Furu KK, Cooper J, Vuong T et al Chemoradiotherapy versus radiotherapy in patients with advanced nasopharyngeal cancer: phase III randomized Intergroup study 0099. J Clin Oncol 1998;16(4):1310-7.

11. Lin JC, Liang WM, Jan JS, Jiang RS, Lin AC. Another way to estimate outcome of advanced nasopharyngeal carcinoma-is concurrent chemoradiotherapy adequate? Int J Radiat Oncol Biol Phys. 2004;60(1):156-64.

12. Balakrishnan U. An additional younger-age peak for cancer of the nasopharynx. Int J Cancer 1975;15:651-7.

13. Shanmugaratnam K, Chan SH, de The G, Goh JE, Khor TH, Simons MJ et al. Histopathology of nasopharyngeal carcinoma: correlations 
with epidemiology, survival rates and other biological characteristics. Cancer 1979;44(3):1029-44.

14. Rickey LM, Olshan AF, George J, Shores CG, Zanation AM, Cannon $\mathrm{T}$ et al. Incidence and survival rates for young blacks with nasopharyngeal carcinoma in the United States. Arch Otolaryngol Head Neck Surg. 2006;132(10):1035-40.

15. Ko J, Lui LT, Sheen T, Lou PJ, Hsu MM. Increased mutagen sensibility in patients with head and neck cancer is less pronounced in patients with nasopharyngeal carcinoma. Arch Otolaryngol Head Neck Surg 1998;124:578-81.

16. Pignon JP, Bourhis J, Domenge C, Designe L. Chemotherapy added to locoregional treatment for head and neck squamous-cell carcinoma: three meta-analyses of updated individual data. MACH-NC Collaborative Group. Meta-Analysis of Chemotherapy on head and neck cancer. Lancet 2000;18(355 Suppl 9208):949-55.

17. Liu LZ, Zhang GY, Xie CM, Liu XW, Ciu CY, Li L. Magnetic resonance imaging of retropharyngeal lymph node metastasis in nasopharyngeal carcinoma: patterns of spread. Int J Radiat Oncol Biol Phys 2006;66(3):721-30.

18. Cengiz M, Ozyar E, Esassolak M, Altun M, Akmansu M, Sen M et al Assessment of quality of life of nasopharyngeal carcinoma patients with EORTC QLQ-C30 and H \& N 35 modules. Int J Radiat Oncol Biol Phys 2005;63(5):1347-53.

19. Rossi A, Molinari R, Boracchi P, Del Vecchio M, Marubini E, Naya $M$ et al. Adjuvant chemotherapy with vincristine, cyclophosphamide and doxorubicin after radiotherap in local-regional nasopharyngeal cancer: results of a 4-year multicenter randomized study. J Clin Oncol 1988;6(9):1401-10

20. Chua DT, Sham JS, Choy D, Lorvidhaya V, Sumitsawan Y, Thongprasert $\mathrm{S}$ et al. Preliminary report of the Asian-Oceanian Clinical Oncology Association randomized trial comparing cisplatin and epirubicin followed by radiotherapy versus radiotherapy alone in the treatment of patients with locoregionally advanced nasopharyngeal carcinoma. Asian-Oceanian Clinical Oncology Association Nasopharynx Cancer Study Group. Cancer 1998;83(11):2270-83.
21. Hoppe RT, Golfinet DR, Bagshaw MA. Carcinoma of the nasopharynx: eighteen years' experience with megavoltage radiation therapy Cancer 1976;37(6):2605-12.

22. Mesic JB, Fletcher GH, Goepfert H. Megavoltage irradiation of epithelial tumors of the nasopharynx. Int J Radiat Oncol Biol Phys $1981 ; 7(4): 447-53$

23. Vikram B, Mishra UB, Strong EW, Manolatos S. Patterns of failure in carcinoma of the nasopharynx: I. Failure at the primary site. Int J Radiat Oncol Biol Phys 1985;11(8):1455-59.

24. Pryzant RM, Wendt CD, Delclos L, Peters LJ. Re-treatment of nasopharyngeal carcinoma in 53 patients. Int J Radiat Oncol Biol Phys 1992;22:941.

25. Wei WI, Ho WK, Cheng AC, Wu X, Li GK, Nicholls J et al. Management of extensive cervical nodal metastasis in nasopharyngeal carcinoma after radiotherapy: a clinicopathological study. Arch Otolaryngol Head Neck Surg 2001;127(12):1457-62.

26. Spano JP, Bresson P, Atlan D, Bourhis J, Pignon JP, Esteban C, et al Nasopharyngeal carcinoma: an update. Eur J Cancer 2003;39(5):212435

27. Hsu MM, Tu SM. Nasopharyngeal carcinoma in Taiwan. Clinical manifestation and results of therapy. Cancer 1983;52(2):362-8.

28. Chua DT, Ma J, Sham JS, Mai HQ, Choy DT, Hong MH, et al. Longterm survival after cisplatin based induction chemotherapy and radiotherapy for nasopharyngeal carcinoma: a pooled data analysis of two phase III trials. J Clin Oncol 2005;23:1118-24.

29. Oh JL, Vokes EE, Kies MS, Mittal BB, Witt ME, Weichselbaum RR, et al. Induction chemotherapy followed by concomitant chemoradiotherapy in the treatment of locoregionally advanced nasopharyngeal cancer. Ann Oncol 2003;14:564-9. 